\title{
Molecular Constants of Aluminum Monohalides - Caveats for Computations of Simple Inorganic Molecules
}

\section{Magdolna Hargittai and Zoltán Varga}

Materials Structure and Modeling Research Group of the Hungarian Academy of Sciences at the Faculty of Chemical Technology and Biotechnology, Budapest University of Technology and Economics, P. O. Box 91, H-1521 Budapest, Hungary

\section{Supporting information}

(for details of computation and references of basis sets, see the paper)

\section{Computed bond length of AlF (in Å)}

$$
r_{\exp }=1.65436(2)^{1}
$$

\begin{tabular}{|c|c|c|c|c|c|c|c|}
\hline \multicolumn{2}{|l|}{ Basis } & \multicolumn{3}{|c|}{$r(\mathrm{Al}-\mathrm{F})$} & \multicolumn{3}{|c|}{$r(\mathrm{Al}-\mathrm{F})$} \\
\hline \multirow[b]{2}{*}{$\mathrm{F}$} & \multirow[b]{2}{*}{$\mathrm{Al}$} & \multicolumn{3}{|c|}{ MP2 } & \multicolumn{3}{|c|}{$\operatorname{CCSD}(\mathrm{T})$} \\
\hline & & default & FC1 & full & default & FC1 & Full \\
\hline cc-pVDZ & cc-pVDZ & 1.710 & 1.710 & 1.710 & 1.710 & 1.711 & 1.711 \\
\hline cc-pVTZ & cc-pVTZ & 1.680 & 1.678 & 1.678 & 1.677 & 1.675 & 1.675 \\
\hline cc-pVQZ & cc-pVQZ & 1.671 & 1.657 & 1.657 & 1.667 & 1.653 & 1.653 \\
\hline cc-pV5Z & cc-pV5Z & 1.668 & 1.660 & 1.660 & 1.663 & 1.656 & 1.655 \\
\hline cc-pV6Z & cc-pV6Z & 1.667 & 1.658 & 1.653 & 1.663 & 1.654 & 1.653 \\
\hline aug-cc-pVDZ & cc-pVDZ & 1.706 & & 1.706 & 1.704 & & 1.704 \\
\hline aug-cc-pVTZ & cc-pVTZ & 1.684 & & 1.677 & 1.680 & & 1.673 \\
\hline aug-cc-pVQZ & cc-pVQZ & 1.673 & & 1.645 & 1.669 & & 1.641 \\
\hline aug-cc-pV5Z & cc-pV5Z & 1.669 & & 1.660 & 1.664 & & 1.655 \\
\hline cc-pwCVDZ & cc-pwCVDZ & 1.693 & 1.695 & 1.695 & 1.693 & 1.695 & 1.695 \\
\hline cc-pwCVTZ & cc-pwCVTZ & 1.662 & 1.657 & 1.657 & 1.658 & 1.653 & 1.653 \\
\hline cc-pwCVQZ & cc-pwCVQZ & 1.665 & 1.658 & 1.658 & 1.660 & 1.654 & 1.654 \\
\hline cc-pwCV5Z & cc-pwCV5Z & 1.665 & 1.658 & 1.659 & 1.662 & 1.655 & 1.654 \\
\hline aug-cc-pwCVDZ & cc-pwCVDZ & 1.692 & 1.690 & & 1.689 & 1.687 & \\
\hline aug-cc-pwCVTZ & cc-pwCVTZ & 1.670 & 1.664 & & 1.666 & 1.660 & \\
\hline aug-cc-pwCVQZ & cc-pwCVQZ & 1.667 & 1.661 & & 1.663 & 1.656 & \\
\hline aug-cc-pwCV5Z & cc-pwCV5Z & 1.667 & 1.660 & & 1.662 & 1.655 & \\
\hline aug-cc-pwCVDZ & aug-cc-pwCVDZ & & 1.690 & & & 1.687 & \\
\hline aug-cc-pwCV5Z & aug-cc-pwCV5Z & & 1.660 & & & 1.655 & \\
\hline
\end{tabular}

\footnotetext{
${ }^{1}$ Wyse, F.C.; Gordy, W.; Pearson, E.F. J. Chem. Phys. 1970, 52, 3887
} 


\section{Computed bond length of AlCl (in Å)}

$$
r_{\exp }=2.13011(3)^{2}
$$

\begin{tabular}{|ll|rrr|rrr|}
\hline Basis & & \multicolumn{3}{|c|}{$r(\mathrm{Al}-\mathrm{Cl})$} & \multicolumn{3}{c|}{$r(\mathrm{Al}-\mathrm{Cl})$} \\
\hline & & \multicolumn{3}{|c|}{ MP2 } & \multicolumn{3}{c|}{ CCSD(T) } \\
Cl & Al & default & FC1 & full & default & FC1 & Full \\
\hline cc-pVDZ & cc-pVDZ & 2.179 & 2.177 & 2.177 & 2.182 & 2.180 & 2.180 \\
cc-pVTZ & cc-pVTZ & 2.152 & 2.145 & 2.145 & 2.154 & 2.148 & 2.148 \\
cc-pVQZ & cc-pVQZ & 2.144 & 2.112 & 2.110 & 2.145 & 2.113 & 2.111 \\
cc-pV5Z & cc-pV5Z & 2.139 & 2.126 & 2.125 & 2.140 & 2.128 & 2.126 \\
cc-pV6Z & cc-pV6Z & 2.138 & 2.124 & 2.121 & 2.139 & 2.126 & 2.123 \\
\hline aug-cc-pVDZ & cc-pVDZ & 2.209 & & 2.205 & 2.212 & & 2.209 \\
aug-cc-pVTZ & cc-pVTZ & 2.159 & & 2.148 & 2.161 & & 2.151 \\
aug-cc-pVQZ & cc-pVQZ & 2.146 & & 2.100 & 2.148 & & 2.101 \\
aug-cc-pV5Z & cc-pV5Z & 2.140 & & 2.124 & 2.142 & & 2.126 \\
\hline cc-pwCVDZ & cc-pwCVDZ & 2.164 & 2.164 & 2.164 & 2.167 & 2.168 & 2.168 \\
cc-pwCVTZ & cc-pwCVTZ & 2.142 & 2.136 & 2.136 & 2.144 & 2.138 & 2.138 \\
cc-pwCVQZ & cc-pwCVQZ & 2.138 & 2.130 & 2.130 & 2.139 & 2.132 & 2.132 \\
cc-pwCV5Z & cc-pwCV5Z & 2.138 & 2.129 & 2.128 & 2.138 & 2.130 & 2.130 \\
\hline aug-cc-pwCVDZ & cc-pwCVDZ & 2.194 & 2.194 & & 2.197 & 2.198 & \\
aug-cc-pwCVTZ & cc-pwCVTZ & 2.149 & 2.143 & & 2.151 & 2.146 & \\
aug-cc-pwCVQZ & cc-pwCVQZ & 2.140 & 2.132 & & 2.142 & 2.134 & \\
aug-cc-pwCV5Z & cc-pwCV5Z & 2.140 & 2.130 & & 2.140 & 2.132 & \\
\hline
\end{tabular}

\footnotetext{
${ }^{2}$ Wyse, F.C.; Gordy, W. J. Chem. Phys. 1972, 56, 2130
} 


\section{Computed bond length of AlBr (in Å)}

$$
r_{\mathrm{e}}=2.29480(3)^{3}
$$

\begin{tabular}{|c|c|c|c|c|c|c|c|c|c|}
\hline Basis & & & $r(\mathrm{Al}$ & $\mathrm{Br})$ & & & $r(\mathrm{~A}$ & & \\
\hline & & & $\mathrm{M}$ & & & & CCS & & \\
\hline $\mathrm{Br}$ & $\mathrm{Al}$ & default & sp & FC1 & full & default & sp & FC1 & Full \\
\hline cc-pVDZ-PP & cc-pVDZ & 2.333 & 2.340 & 2.331 & 2.331 & 2.338 & 2.343 & 2.336 & 2.336 \\
\hline cc-pVTZ-PP & cc-pVTZ & 2.307 & 2.318 & 2.301 & 2.301 & 2.311 & 2.320 & 2.306 & 2.306 \\
\hline cc-pVQZ-PP & cc-pVQZ & 2.296 & 2.310 & 2.261 & 2.258 & 2.301 & 2.312 & 2.265 & 2.262 \\
\hline cc-pV5Z-PP & cc-pV5Z & 2.291 & 2.306 & 2.285 & 2.282 & 2.296 & 2.309 & 2.290 & 2.287 \\
\hline aug-cc-pVDZ-PP & cc-pVDZ & 2.351 & 2.353 & & 2.352 & 2.355 & 2.357 & & 2.355 \\
\hline aug-cc-pVTZ-PP & cc-pVTZ & 2.313 & 2.323 & & 2.303 & 2.318 & 2.326 & & 2.309 \\
\hline aug-cc-pVQZ-PP & cc-pVQZ & 2.297 & 2.311 & & 2.253 & 2.302 & 2.314 & & 2.257 \\
\hline aug-cc-pV5Z-PP & сc-pV5Z & 2.292 & 2.307 & & 2.282 & 2.297 & 2.310 & & 2.287 \\
\hline cc-pVDZ-PP & cc-pwCVDZ & 2.324 & 2.330 & 2.326 & 2.326 & 2.328 & 2.334 & 2.330 & 2.330 \\
\hline cc-pVTZ-PP & cc-pwCVTZ & 2.300 & 2.311 & 2.297 & 2.296 & 2.304 & 2.314 & 2.301 & 2.301 \\
\hline cc-pVQZ-PP & cc-pwCVQZ & 2.288 & 2.305 & 2.282 & 2.282 & 2.292 & 2.308 & 2.287 & 2.286 \\
\hline cc-pV5Z-PP & cc-pwCV5Z & 2.290 & 2.305 & 2.283 & 2.283 & 2.295 & 2.308 & 2.288 & 2.288 \\
\hline cc-pwCVDZ-PP & cc-pwCVDZ & 2.320 & & 2.322 & 2.321 & 2.327 & & 2.328 & 2.328 \\
\hline cc-pwCVTZ-PP & cc-pwCVTZ & 2.301 & & 2.297 & 2.297 & 2.307 & & 2.303 & 2.303 \\
\hline cc-pwCVQZ-PP & cc-pwCVQZ & 2.295 & & 2.289 & 2.289 & 2.301 & & 2.295 & 2.295 \\
\hline aug-cc-pwCVDZ-PP & cc-pwCVDZ & 2.351 & & 2.352 & & 2.356 & & 2.356 & \\
\hline aug-cc-pwCVTZ-PP & cc-pwCVTZ & 2.307 & & 2.302 & & 2.313 & & 2.309 & \\
\hline aug-cc-pwCVQZ-PP & cc-pwCVQZ & 2.297 & & 2.291 & & 2.303 & & 2.297 & \\
\hline aug-cc-pwCVDZ-PP & aug-cc-pwCVDZ & & & 2.347 & & & & 2.352 & \\
\hline aug-cc-pwCVQZ-PP & aug-cc-pwCVQZ & & & 2.290 & & & & 2.296 & \\
\hline SDB-cc-pVDZ & cc-pVDZ & 2.345 & & & 2.344 & 2.348 & & & 2.347 \\
\hline SDB-cc-pVTZ & cc-pVTZ & 2.311 & & & 2.308 & 2.315 & & & 2.312 \\
\hline SDB-cc-pVQZ & cc-pVQZ & 2.304 & & & 2.268 & 2.307 & & & 2.271 \\
\hline
\end{tabular}

\footnotetext{
${ }^{3}$ Wyse, F.C.; Gordy, W. J. Chem. Phys. 1972, 56, 2130
} 


\section{Computed bond length of AlI (in Å)}

$$
r_{\exp }=2.53709(3)^{4}
$$

\begin{tabular}{|c|c|c|c|c|c|c|c|c|c|}
\hline \multicolumn{2}{|l|}{ Basis } & \multicolumn{4}{|c|}{$r(\mathrm{Al}-\mathrm{I})$} & \multicolumn{4}{|c|}{$r(\mathrm{Al}-\mathrm{I})$} \\
\hline \multirow[b]{2}{*}{ I } & \multirow[b]{2}{*}{$\mathrm{Al}$} & \multicolumn{4}{|c|}{ MP2 } & \multicolumn{4}{|c|}{ CCSD(T) } \\
\hline & & default & sp & FC1 & full & default & $\mathrm{sp}$ & FC1 & full \\
\hline cc-pVDZ-PP & cc-pVDZ & 2.576 & 2.583 & 2.574 & 2.574 & 2.578 & 2.586 & 2.578 & 2.578 \\
\hline cc-pVTZ-PP & cc-pVTZ & 2.547 & 2.561 & 2.547 & 2.547 & 2.552 & 2.565 & 2.552 & 2.546 \\
\hline cc-pVQZ-PP & cc-pVQZ & 2.536 & 2.554 & 2.502 & 2.499 & 2.542 & 2.558 & 2.508 & 2.505 \\
\hline сc-pV5Z-PP & cc-pV5Z & 2.528 & 2.552 & 2.520 & 2.518 & 2.534 & 2.556 & 2.527 & 2.525 \\
\hline aug-cc-pVDZ-PP & cc-pVDZ & 2.599 & 2.603 & & 2.595 & 2.604 & 2.607 & & 2.600 \\
\hline aug-cc-pVTZ-PP & cc-pVTZ & 2.556 & 2.565 & & 2.546 & 2.561 & 2.569 & & 2.551 \\
\hline aug-cc-pVQZ-PP & cc-pVQZ & 2.539 & 2.556 & & 2.500 & 2.546 & 2.560 & & 2.506 \\
\hline aug-cc-pV5Z-PP & cc-pV5Z & 2.529 & 2.553 & & 2.519 & 2.535 & 2.557 & & 2.526 \\
\hline cc-pwCVDZ-PP & cc-pwCVDZ & 2.560 & & 2.560 & 2.560 & 2.569 & & 2.569 & 2.569 \\
\hline cc-pwCVTZ-PP & cc-pwCVTZ & 2.535 & & 2.531 & 2.531 & 2.545 & & 2.540 & 2.541 \\
\hline cc-pwCVQZ-PP & cc-pwCVQZ & 2.530 & & 2.523 & 2.523 & 2.539 & & 2.534 & 2.534 \\
\hline aug-cc-pwCVDZ-PP & cc-pwCVDZ & 2.577 & & 2.577 & & 2.586 & & 2.586 & \\
\hline aug-cc-pwCVTZ-PP & cc-pwCVTZ & 2.539 & & 2.535 & & 2.549 & & 2.545 & \\
\hline aug-cc-pwCVQZ-PP & cc-pwCVQZ & 2.530 & & 2.525 & & 2.541 & & 2.535 & \\
\hline SDB-cc-pVDZ & cc-pVDZ & 2.562 & & & 2.561 & 2.565 & & & 2.564 \\
\hline SDB-cc-pVTZ & cc-pVTZ & 2.555 & & & 2.550 & 2.559 & & & 2.555 \\
\hline SDB-cc-pVQZ & cc-pVQZ & 2.548 & & & 2.517 & 2.552 & & & 2.521 \\
\hline
\end{tabular}

\footnotetext{
${ }^{4}$ Wyse, F.C.; Gordy, W. J. Chem. Phys. 1972, 56, 2130
} 